\title{
ORGANIZING COMMITTEES
}

The Congress Chairmen :

- Diane Goéré (Paris, France)

- Olivier Glehen (Lyon, France)

- Marc Pocard (Paris, France)

The Local Organizing Committee :

- Jean-Marc Bereder (Nice, France)

- Frédéric Bideau (Montpellier, France)

- Valérie Billard (Paris, France)

- Jean-Marc Classe (Saint Herblain, France)

- Antony Dohan (Paris, France)

- Clarisse Eveno (Paris, France)

- Gilles Freyer (Lyon, France)

- Maximiliano Gelli (Paris, France)

- Laurence Gladieff (Tououse, France)

- Rea Lo Dico (Paris, France)

- Guillaume Passot (Lyon, France)

- François Quenet (Montpellier, France)

- Pascal Rousset (Lyon, France)

- Olivia Sgarbura (Montepellier, France)
The Scientific Committee :

- Paul H Sugarbaker (USA)

- David Barlett (USA)

- Wim Ceelen (Belgium)

- Marcello Deraco (Italy)

- Jesus Esquivel (USA)

- Ignace de Hingh (The Netherlands)

- Joji Kitayama (Japan)

- Yan Li (China)

- Brendan Moran (UK)

- Santiago Gonzalez Moreno (Spain)

- David Morris (Australia)

- Aviram Nissan (Israel)

- Pompiliu Piso (Germany)

- Claudio Quadros (Brazil)

- Beate Rau (Germany)

- Vic Verwaal (Denmark)

- Yutaka Yonemura (Japan) 